\title{
History and Mechanism for Treatment of Intracerebral Hemorrhage with Scalp Acupuncture
}

\author{
Zhe Liu, ${ }^{1}$ Ling Guan,, ${ }^{2}$ Yan Wang, ${ }^{3}$ Cheng-Long Xie, ${ }^{3}$ Xian-Ming Lin, ${ }^{1}$ and Guo-Qing Zheng ${ }^{3}$ \\ ${ }^{1}$ The Third Clinical College of Zhejiang Chinese Medical University, Hangzhou 310005, China \\ ${ }^{2}$ Department of Acupuncture and Moxibustion, General Hospital of the Chinese People's Liberation Army, Beijing 100853, China \\ ${ }^{3}$ Center of Neurology and Rehabilitation, The Second Affiliated Hospital of Wenzhou Medical College, Wenzhou 325027, China
}

Correspondence should be addressed to Guo-Qing Zheng, gq_zheng@sohu.com

Received 23 November 2011; Accepted 14 December 2011

Academic Editor: Gerhard Litscher

Copyright ( 2012 Zhe Liu et al. This is an open access article distributed under the Creative Commons Attribution License, which permits unrestricted use, distribution, and reproduction in any medium, provided the original work is properly cited.

\begin{abstract}
Intracerebral hemorrhage (ICH) is an important public health problem with high rates of mortality, morbidity, and disability, but no clinically proven treatment strategy is available to date. Scalp acupuncture (SA) refers to a therapy for treating diseases by needling and stimulating the specific areas of the scalp. The evidence from clinical studies suggested that SA therapy may produce significant benefits for patients with acute ICH. However, the therapeutic mechanisms are yet not well addressed. Therefore, in this paper, we provide a comprehensive overview on the history and mechanisms of SA therapy on acute ICH. Although SA has been practiced for thousands of years in China and could date back to $5 \mathrm{BC}$, SA therapy for acute ICH develops only in the recent 30 years. The possible mechanisms associated with the therapeutic effects of SA on ICH include the influence on hematoma, brain edema, and blood brain barrier, the products released from haematoma, the immune and inflammatory reaction, focal perihemorrhagic hypoperfusion and hemorheology, neuroelectrophysiology, and so on. At last, the existence of instant effect of SA on acute ICH and its possible mechanisms are presented.
\end{abstract}

\section{Introduction}

Stroke is the second most common cause of death after heart attacks and the major cause of disability in the Western world [1], which may soon become the leading cause of death worldwide [2]. Primary intracerebral hemorrhage (ICH) is a devastating stroke with a higher death and disability rate compared to ischemic stroke and accounts for $10 \sim 15 \%$ of all strokes. However, no effective therapy to date could be established in a large trial and its outcome remains poor [3]. Furthermore, more than two-thirds of the burden of global stroke occurs in developing (low- and middle-income) countries [4]. Among developing countries, China has the largest population, about one-fifth of the world's population. Simultaneously, the incidence of hemorrhagic stroke in China was generally higher than that in the Western countries [5]. Because high proportions of ICH occur in developing countries, the setting of new research directions is sorely needed [6].

The most appreciable distinction between China and the West in treating stroke is the use of traditional Chinese medicine (TCM) which includes herbal medicine, acupuncture, and other nonmedication therapies $[7,8]$. TCM has played an important role in the medical care of stroke patients for thousands of years [9]. Currently, various unconventional local therapeutic traditions are widely used for stroke treatment in China, although Level I, class A evidence of effective treatment is lacking [10]. Acupuncture therapy, which is a major therapeutic method in Chinese traditional healing systems, has also been used in stroke therapy for a long time. Scalp acupuncture (SA), also called scalp acupuncture therapy, cranial needling therapy, and scalp acupoints penetration therapy, is developed based on the traditional acupuncture science, modern anatomy, neuroz physiology, and bioholographic theory. SA belongs to micropuncture system, in which filiform needle is used to penetrate specific stimulation areas on scalp. It is often applied for treatment of central nervous system disorders and various acute or chronic pains and has achieved specific curative effects [11]. Particularly, SA therapy was used in the treatment of both ischemic stroke and hemorrhagic stroke with 
TABLE 1: Brief History of the Development of Scalp Acupuncture.

\begin{tabular}{|c|c|c|}
\hline Year & Author and/or work & Contribution \\
\hline $5 \mathrm{BC}$ & Bian Que's biography & $\begin{array}{l}\text { The earliest medical record for the application of SA } \\
\text { therapy in China }\end{array}$ \\
\hline $221 \mathrm{BC}-220 \mathrm{AD}$ & Huangdi Neijing (Huangdi's internal classics) & Establishment of the TCM theory basis for SA therapy. \\
\hline $220 \mathrm{AD}-1950 \mathrm{AD}$ & $\begin{array}{l}\text { There were rich records of SA therapy in the medical } \\
\text { books. }\end{array}$ & $\begin{array}{l}\text { SA therapy developed through different generations, } \\
\text { but it still belonged to the traditional acupuncture } \\
\text { system. }\end{array}$ \\
\hline 1950’s & SA became one part of micropuncture systems. & SA began to form up an independent system. \\
\hline 1970’s & $\begin{array}{l}\text { Jiao Shunfa SA therapy, Fang Yunpeng SA therapy, Zhu } \\
\text { Longyu SA therapy, and Zhu Minqing SA therapy, } \\
\text { Yamamoto et al. new SA }\end{array}$ & $\begin{array}{l}\text { SA therapy developed rapidly and has formed various } \\
\text { scalp acupoint nomenclature systems. }\end{array}$ \\
\hline 1980's-1990's & $\begin{array}{l}\text { WHO Scientific Group on International Acupuncture } \\
\text { Nomenclature }\end{array}$ & $\begin{array}{l}\text { The international standard SA lines were approved by } \\
\text { scientific group of WHO. }\end{array}$ \\
\hline
\end{tabular}

SA, Scalp Acupuncture. WHO, World Health Organization.

good therapeutic effects in China [12]. Recently, a Metaanalysis done by our group showed that SA therapy could be able to improve neurological deficit in acute hypertensive ICH patients [13]. However, the mechanisms of the therapeutic effects of SA on acute ICH are not well addressed. Therefore, in this paper, we provide a comprehensive overview on the history and mechanisms of SA therapy on acute ICH.

\section{A Brief History of Scalp Acupuncture and Its Application in Acute Intracerebral Hemorrhage}

SA therapy has been practiced for thousands of years. Doctors of ancient time had already known the importance of scalp acupoints from practice, but SA develops very fast only in recent decades. A brief history of the development of SA has been summarized in Table 1. SA is originated from cranial acupuncture for disease treatment in ancient China. In the historical records-Bian Que's Biography, there may have the earliest medical record for the application of SA therapy in China. It was said that in $5 \mathrm{BC}$, Bian Que cured a patient (Guo state's crown prince) suffering from Shi jue (false death) with acupuncture on Baihui (GV 20) [14]. Huangdi Neijing (Huangdi's Internal Classics, HIC) established the TCM theory basis for SA therapy. In this theory, head is where all Yang-meridians meeting together and is the house of the original spirit. Head is also where convergence between meridian/channel qi and blood happens. Based on the theory "starting and terminal point of meridian/channel" and the theory "manifestation and root cause of meridian/channel", scalp acupoints and body acupoints are closely related with extremity acupoints. Brain is the sea of marrow and is the master of Zang-fu organs and meridian/channel functional activities. Acupoints on the head can regulate function of Yin-yang, Qi-blood, and Zangfu organs. This is the main theory basis for SA therapy. There are also many records of SA treatment cases in HIC. There are more than 50 parts in Zhenjiujiayijing (A$B$ Classic of Acupuncture and Moxibustion) of Jin Dynasty recording treatment of disease through head acupoints, for example, the diseases of head, face and the five sensory organs, and psychiatry and neurologic diseases, and so forth. Afterward, SA therapy gradually developed through different generations and the records were rich in medical books. Mister Cheng Danan (1899-1957) found the first professional journal of acupuncture-Journal of Acupuncture in October 1933. Reports are published about curing diseases through the combination of acupoints on the body and acupoints on scalp like Baihui (GV 20), Fengfu (GV 16), Shenting (GV 24), Shangxing (GV 23), Fengchi (GB 20), and Shuaigu (GB 8). But the records are rare about treating disease only with needling scalp acupoints, and also the level of curing diseases on all part of the body with scalp acupoints has not reached. Therefore, it still belonged to the traditional acupuncture system. The common prescriptions usually consisted of both scalp acupoints and distal-limb points in combination. It was mainly used for the treatment of mind illness, head, face and ear-nose-throat diseases, and so forth and did not form up an independent system.

From the 1950's, acupuncture experts began to observe the relationship between body locations of diseases with the corresponding subscalp places, and to define the associativity and effectiveness of the inspiration of micropuncture system such as auricular acupuncture, nose acupuncture, and eye acupuncture, for disease treatment. These kinds of microacupuncture points are distributed in different regions of the body as point(s) or line(s). Needling on the points of one region is able to treat problems of the other regions as well as problems of its own. The application of SA therapy increased obviously since then, and the clinical experience became richer. For example, Fang Yunpeng in Shanxi at the end of 1950's and Tang Yansong in Shanghai at the end of 1960 's began to apply scalp acupuncture for curing diseases, and gradually improved this method.

In the early 1970's, SA was separated from traditional acupuncture system under the influence of neuroanatomy, neurophysiology, and bioholographic principle of modern medicine. New theories and needling methods are formed up and significant curative effects have achieved in the treatment of brain diseases. Acupuncture society put great emphasis on 
SA study and has formed up various scalp acupoint nomenclature systems. SA was gradually developed as an independent and complete acupuncture system. In 1971, Jiao Shunfa from Shanxi province in China first established a modern acupuncture technique combining traditional needling methods with western medical knowledge of representative areas of the cerebral cortex, including anatomy, physiology, pathology, and neurology [15]. Jiao's SA is based on the Western medical theory of nerve system and stimulates specific areas such as motor area, sensory area, and praxis area. The scalp areas corresponding to the functional locations of cerebral cortex are taken as the stimulating areas in SA for the treatment of diseases. Later, Fang Yunpeng (Shanxi) SA therapy was published in 1976 and Zhu Longyu (Shanxi) cranial acupuncture therapy published in 1979 in China [16]. The other SA therapy founders include Zhang Mingjiu (Nanjing), Tang Songyan (Shanghai), and Lin Xuejian (Shanghai) et al. Each of them proposed different diagrams and groupings of scalp acupuncture points. For example, some divided the scalp into zones or regions, while some focused on points or lines.

In 1970's, some papers were published in English journals which introduced SA therapy in the treatment of brain diseases [17-19]. In America, Dr. Zhu Minqing (Beijing) began developing his SA method in the 1970's and brought his practice to the United States and created the Chinese Scalp Acupuncture Center of USA in 1991, San Francisco. His practice now expands to Zhu's Acupuncture Medical \& Neurology Center, in San Jose, CA. In 1992, he published an English-language book on his methods: Zhu's Scalp Acupuncture [20]. According to Dr. Zhu, Baihui (GV-20) is the basis for all of the scalp points. In Zhu's SA therapy, there are three main zones (designated the Eding zone, Dingzhen zone and Dingnie zone) subdivided into a total of 11 portions and three secondary zones, each divided into two portions (designated Epang 1, Epang 2, front zone of Dingjie, back zone of Dingjie, Niehou and Nieqian). In Japan, based on what he identified as somatotopes, Dr. Toshikatsu Yamamoto has developed what is known as Yamamoto New Scalp Acupuncture (YNSA). YNSA was presented to the world for the first time in 1973. It involves point selection through abdominal or neck test zone palpation, with extremely accurate fine needling of the appropriate scalp points [21]. In 1998, a book named Yamamoto new SA therapy was published [22]. Although there are many differences between Japanese and Chinese SA therapy like in the theoretical basis, the stimulating sites, and the names of acupoints, they still have many common aspects in clinical indications and manipulating methods.

As SA therapy was widely used in China and its effectiveness was verified through experience, it was gradually accepted and became a commonly used therapy in many countries around the world. The standardization of the names of acupoints was thus very necessary and urgent, so it was put on the agenda. In 1983, the World Health Organization (WHO) Western Pacific Ocean Region Committee entrusted China Acupuncture Association to prepare the scheme of Standard Nomenclature of SA lines. After the discussion in the standardization working group in 1984, 1985, and 1987, consensus of opinion has been reached and named as $A$ Proposed Standard International Acupuncture Nomenclature: 3.6 Scalp acupuncture lines. This scheme was formally adopted in a science group meeting hold by WHO in 1989 and was published in 1991 [23]. This nomenclature was formulated according to the domains on the meridians and combined with the traditional penetration puncture technique (one needle penetrate two or three points). The nomenclature consists of three parts: the codes, Chinese character names, and Chinese spelling names. The codes include initials of microsystem and scalp point (MS) and the corresponding serial number. There are $14 \mathrm{SA}$ point lines in all spreading in 4 regions (Table 2). The nomenclature is a great contribution to the development and popularization of SA therapy and to the international academic exchange of SA study. Acupuncture can be performed manually (manual acupuncture) or electrically (electric acupuncture), the former including penetration needling of scalp acupoints. Insert the needle subcutaneously, $0.35 \mathrm{~mm}-0.45 \mathrm{~mm}$ in diameter and $40 \mathrm{~mm}-70 \mathrm{~mm}$ in length, with an angle of $15 \sim 30^{\circ}$. Twirl the needle continuously or repeatedly quickly. Electraacupuncture is applicable, but the intensity should be set appropriately. During the manipulation, blood vessels should be kept away. After plucking the needle, press the pinhole to avoid subcutaneous hematoma [24].

Therefore, SA has many schools or systerms. For example, in the SA school of Dr. Jiao, Fang et al., the theory base is mainly cerebral physiology and anatomy. The school of international standard scalp acupuncture lines is largely based on meridian theory and acupoints on the head. In a word, SA is not a single system, but a multiplicity of systems still in development.

SA therapy for acute ICH develops only in the recent 30 years (Table 3). Until 1980's, some study groups started to explore SA therapy for acute ICH. In 1991, Dong's group from Heilung-kiang University of TCM first published the clinical trial that applied scalp penetration acupuncture in the treatment of acute ICH [25] and subsequent series of studies showed that SA therapy for acute ICH were generally safe and achieved significant benefits. Based on cerebral projection on the scalp and general regulation theory, penetration needling of scalp acupoints is used in SA. Because other acupoints are also stimulated through needling one acupoint and other meridians are simultaneously stimulated through one meridian, penetration needling can synthetically modulate function of brain and body, so as to reach the goal of treatment. In 1999, a well-designed clinical trial was conducted by Li et al. [26], suggesting that SA was safe and effective in treating acute ICH. In 2006, our group published the first paper entitled "Literature foundation of scalp-acupuncture treatment of cerebral hemorrhage and its recent progress in clinical research" [27]. In 2009, our group put forward some new ways of thinking and new methodological standards on stroke study using SA, and this research may provide methodological reference for future study on the mechanism [12]. Recently, our group conducted the first Meta-analysis of SA therapy for acute hypertensive $\mathrm{ICH}[13]$. 
TABLE 2: Nomenclature of scalp acupuncture lines by scientific group of WHO.

\begin{tabular}{|c|c|c|c|c|}
\hline Regions & English name and location & Pinyin name & Han character name & Alphan-umeric code \\
\hline \multirow{4}{*}{ Forehead region } & $\begin{array}{l}\text { Middle line of forehead } 1 \text { cun } \\
\text { from GV } 24 \text { straight down along } \\
\text { the meridian. }\end{array}$ & Ézhongxiàn & 額中線 & MS1 \\
\hline & $\begin{array}{l}\text { Lateral line } 1 \text { of forehead } 1 \text { cun } \\
\text { from BL3 straight down along } \\
\text { the meridian. }\end{array}$ & Épángxiàn I & 額旁1線 & MS2 \\
\hline & $\begin{array}{l}\text { Lateral line } 2 \text { of forehead } 1 \text { cun } \\
\text { from GB15 straight down along } \\
\text { the meridian. }\end{array}$ & Épángxiàn II & 額旁2線 & MS3 \\
\hline & $\begin{array}{l}\text { Lateral line } 3 \text { of forehead } 1 \text { cun } \\
\text { from the point } 0.75 \text { cun medial } \\
\text { to ST8 straight down. }\end{array}$ & épángxiàn III & 額旁3線 & MS4 \\
\hline \multirow{6}{*}{ Parietal region } & $\begin{array}{l}\text { Middle line of vertex from GV } 20 \\
\text { to GV } 21 \text { along the midline of } \\
\text { head. }\end{array}$ & Dingzhongxiàn & 頂中線 & MS5 \\
\hline & $\begin{array}{l}\text { Anterior oblique line of } \\
\text { vertex-temporal from }\end{array}$ & & & \\
\hline & $\begin{array}{l}\text { qiánshéncong 前神衝 (one of } \\
\text { the four acupuncture points } \\
\text { collectively designated as } \\
\text { Ex-HN1, 1 cun anterior to GV } \\
\text { 20) obliquely to GB6. }\end{array}$ & Dingniè qiánxiéxiàn & 丁頁顳前斜線 & MS6 \\
\hline & $\begin{array}{l}\text { Posterior oblique line of } \\
\text { vertex-temporal from GV } 20 \\
\text { obliquely to GB7. }\end{array}$ & Dingniè hòuxiéxiàn & 丁頁顳後斜線 & MS7 \\
\hline & $\begin{array}{l}\text { Lateral line } 1 \text { of vertex } 1.5 \text { cun } \\
\text { lateral to middle line of vertex, } \\
1.5 \text { cun from BL6 backward } \\
\text { along the meridian. }\end{array}$ & Dingpángxiàn I & 頂旁1線 & MS8 \\
\hline & $\begin{array}{l}\text { Lateral line } 2 \text { of vertex } 2.25 \text { cun } \\
\text { lateral to middle line of vertex, } \\
1.5 \text { cun from GB17 backward } \\
\text { along the meridian. }\end{array}$ & Dingpángxiàn II & 頂旁2線 & MS9 \\
\hline \multirow[t]{2}{*}{ Temporal region } & $\begin{array}{l}\text { Anterior temporal line from GB4 } \\
\text { to GB6. }\end{array}$ & Nièqiánxiàn & 顳前線 & MS10 \\
\hline & $\begin{array}{l}\text { Posterior temporal line from } \\
\text { GB8 to GB7. }\end{array}$ & Nièhòuxiàn & 顳後線 & MS11 \\
\hline \multirow{3}{*}{ Occipital region } & $\begin{array}{l}\text { upper-middle line of occiput } \\
\text { from GV } 18 \text { to GV } 17\end{array}$ & $\begin{array}{l}\text { Zhenshàng } \\
\text { zhèngzhongxiàn }\end{array}$ & 枕上正中線 & MS12 \\
\hline & $\begin{array}{l}\text { Upper-lateral line of occiput } \\
0.5 \text { cun lateral and parallel to } \\
\text { upper-middle line of occiput. }\end{array}$ & Zhenshàng pángxiàn & 枕上旁線 & MS13 \\
\hline & $\begin{array}{l}\text { Lower-lateral line of occiput } \\
2 \text { cun from BL9 straight down. }\end{array}$ & Zhenxià pángxiàn & 枕下旁線 & MS14 \\
\hline
\end{tabular}

\section{Mechanisms for Treatment of ICH with Scalp Acupuncture}

The main pathophysiological phases after ICH consist of direct pathological damage of hematoma information and secondary pathological damage, including early hematoma enlargement and perihematoma oedema due to the breakdown products released from the hematoma. Qureshi et al. [28] has summarized that a secondary cascade of neural injury initiated by $\mathrm{ICH}$ is started by products of coagulation and haemoglobin breakdown; products released by activated microglia such as oxygen free radicals, matrix metallopeptidase (MMP), complement factors, tumour necrosis factor (TNF)- $\alpha$, interleukin (IL)- $1 \beta$, and their expression in aquaporin (AQP)-4 astrocytes; breakdown of connective tissue in blood-brain barrier (BBB); expression of adhesion molecules that induce breakdown of the BBB, vasogenic oedema, and apoptosis in neurons and glia. The possible mecha- 
TABLE 3: Brief history of SA therapy for acute intracerebral hemorrhage.

\begin{tabular}{|c|c|c|}
\hline Year & Author and/or work & Contribution \\
\hline 1980’s & Some study groups & Began to explore SA therapy for acute ICH. \\
\hline 1992 & Dong et al. 1992 [25] & First clinical trial of the SA therapy for acute ICH. \\
\hline 1999 & Li et al. 1999 [26] & $\begin{array}{l}\text { A well-designed clinical trial to study the therapeutic effect of SA in treating } \\
\text { acute ICH and its mechanisms. }\end{array}$ \\
\hline 2006 & Zheng et al. 2006 [27] & $\begin{array}{l}\text { A first review of this field entitled "Literature foundation of scalp-acupuncture } \\
\text { treatment of ICH and its recent progress in clinical research". }\end{array}$ \\
\hline 2009 & Zheng 2009 [12] & $\begin{array}{l}\text { To provide methodological reference for future mechanism study on stroke } \\
\text { study using SA. }\end{array}$ \\
\hline 2010 & Zheng et al. 2010 [13] & A first Meta-analysis of SA therapy for acute hypertensive ICH. \\
\hline
\end{tabular}

SA, Scalp Acupuncture. ICH, intracerebral hemorrhage.

TABLE 4: Possible mechanisms for treatment of acute ICH with scalp acupuncture.

\begin{tabular}{|c|c|}
\hline Possible targets & Possible mechanisms \\
\hline $\begin{array}{l}\text { Influence on hematoma, brain edema and, blood } \\
\text { brain barrier }\end{array}$ & $\begin{array}{l}\text { Improve the absorption rate of hematoma, reduce brain edema, diminish } \\
\text { cerebral vessel permeability, and promote reparation for BBB damage. }\end{array}$ \\
\hline Influence on the products released from haematoma & Inhibit the expression of MMP-9, AQP-4, and PAR- 1. \\
\hline Influence on the immune and inflammatory reaction & $\begin{array}{l}\text { Inhibit the expression of IL- } 1 \beta \text {, decrease serum IL- } 6 \text { level, inhibit TNF- } \alpha \\
\text { expression, and promote HSP70 expression. Increase CD } 3 \text { and CD4/CD } 8 \\
\text { level, while decreasing CD } 8 \text { level. }\end{array}$ \\
\hline $\begin{array}{l}\text { Influence on focal perihemorrhagic hypoperfusion and } \\
\text { hemorheology }\end{array}$ & $\begin{array}{l}\text { improve energy metabolism, upregulate the activity of hondriosome } \\
\text { (Succinate dehydrogenase and } \mathrm{Na}^{+}-\mathrm{K}^{+} \text {-ATPase), reduce the accumulation of } \\
\text { lactate acid, delay Glut-1 expression, lower plasma ET level and NSE level, and } \\
\text { heighten CGRP level. }\end{array}$ \\
\hline Influence on neuroelectrophysiology & $\begin{array}{l}\text { Relieve the inhibitive generalization of the whole brain neuron function, have } \\
\text { bidirectional and beneficial regulation effect on electric activity of } \\
\text { pain-reaction neurons, and recover abnormal SEPs. }\end{array}$ \\
\hline Others & $\begin{array}{l}\text { Promote the NCF gene expression, promote the expression of endogenous } \\
\text { GDNF, lower the level of serum S-100B, and promote the level of IGF- } 1 \text {. }\end{array}$ \\
\hline
\end{tabular}

BBB, blood-brain barrier. MMP-9, matrix metallopeptidase-9. AQP-4, aquaporin-4. PAR-1, protease-activated receptor-1. IL, interleukin. TNF- $\alpha$, tumour necrosis factor $\alpha$. HSP70, heat shock protein 70. Glut-1, glucose transporter-1. ET, endothelin. NSE, neuron specific enolase. CGRP, calcitonin gene-related peptide. SEPs, somatosensory-evoked potentials. NCF, nerve growth factor. GDNF, glial cell line-derived neurotrophic factor. IGF-1, insulin-like growth factor 1 .

nisms for SA therapy in acute ICH were summarized in Table 4, based on the recent researches.

\section{Influence on Hematoma, Brain Edema and Blood Brain Barrier}

Early brain odema volume relative to hematoma volume makes the greatest contribution to clinical outcome [29]. In ICH patients, head CT scan showed that acupuncture on Baihui (GV 20) penetrating Taiyang (EX-HN5) improved the absorption rate of hematoma [30]. In ICH rats, acupuncture on Baihui (GV 20) penetrating Qubin (GB 7) reduced brain edema [31]. Similarly, acupuncture on Baihui (GV 20), Shuigou (GV 26), Hegu (LI 4), and Taichong (LR 3) could accelerate the extenuation of brain edema and diminish cerebral vessel permeability and brain tissue damage in acute $\mathrm{ICH}$ rats [32]. BBB disruption is a hallmark of $\mathrm{ICH}$-induced brain injury [33]. Electric acupuncture on Baihui (GV 20) penetrating Taiyang (EX-HN5) could promote reparation of BBB damage after acute ICH in rats [34].

\section{Influence on the Products Released from Haematoma}

In recent years, more researches focused on the role of local hematoma in the formation of brain edema after ICH. The products released from the intracerebral haematoma or components of blood itself may create the material basis of brain edema. Zhang et al. $[35,36]$ reported that the therapeutic effects of Baihui (GV 20) penetrating Qubin (GB 7) may be through the way of inhibiting the expression of endogenous MMP-9 and AQP-4 in acute ICH rat. Thrombin plays an important role in progression of oedema and injury to nerve cells and axons surrounding the haematoma [37]. Thrombin regulates cellular functions in a large variety of 
cells through protease-activated receptors (PARs). Chi et al. [38] showed that acupuncture on Baihui (GV 20) penetrating Qubin (GB 7) can effectively inhibit the expression of PAR-1 in brain tissue of the acute ICH rat.

\section{Influence on the Immune and Inflammatory Reaction}

When ICH occurs, blood components including erythrocytes, leukocytes, macrophages, and plasma proteins (thrombin, plasmin, etc.) immediately enter the brain. An inflammatory cascade response follows that involves enzyme activation, mediator release, inflammatory cell migration, glial activation, brain tissue breakdown, and repair [39]. The therapy of Baihui (GV 20) penetrating Qubin (GB 7) can inhibit the expression of IL- $1 \beta$ in brain tissue surrounding the hematoma of acute $\mathrm{ICH}$ rat [40]. Furthermore, scalp needling led to rapid decrease of serum IL- 6 content and improved nervous function in the patients of acute ICH [41]. Thirdly, acupuncture on Baihui (GV 20) penetrating Taiyang (EX-HN5) has an obvious inhibitory effect on the immuneinflammatory reaction mediated by TNF- $\alpha$ expression in acute ICH rats [42]. Fourthly, acupuncture on Baihui (GV 20) penetrating Qubin (GB 7) promoted heat shock protein 70 (HSP70) mRNA expression in brain tissue in acute ICH rats [43]. At last, acupuncture on Baihui (GV 20) penetrating Taiyang (EX-HN5) could significantly improve immune function in acute ICH patients. CD3 and CD4/CD8 level increased, while CD8 level decreased. Immune function of patient with acute ICH could benefit greatly from SA therapy [44].

\section{Influence on Focal Perihemorrhagic Hypoperfusion and Hemorheology}

Cerebral ischemia has been proposed as a mechanism contributing to secondary neuronal injury after ICH, but focal perihemorrhagic hypoperfusion is recently considered as a consequence of reduced metabolic demand, that is, diaschisis rather than a sign of ischemia [45]. Electric acupuncture on Baihui (GV 20) penetrating Taiyang (EX-HN5) improved mitochondrial energy metabolism in brain, upregulated the activity of hondriosome (Succinate dehydrogenase and $\mathrm{Na}^{+}$$\mathrm{K}^{+}$-ATPase), and reduced the accumulation of lactate acid in the perihematoma tissue of ICH rats [46]. Scalp electroacupuncture on Baihui (GV 20) penetrating Taiyang (EXHN5) in acute ICH rat can delay glucose transporter-1 (Glut-1) and Glut-1 mRNA expressions at brain tissue surrounding the hematoma [47]. Acupuncture on Baihui (GV 20) penetrating Taiyang (EX-HN5) lowered plasma endothelin (ET) level and neuron specific enolase (NSE) level and heightened calcitonin gene-related peptide (CGRP) level in the patient of acute ICH. These effects may regulate the function of blood vessel, reduce cerebral injury, and improve the neurological function of patients with acute $\mathrm{ICH}[48,49]$.

\section{Influence on Neuroelectrophysiology}

Electrical activity of brain is a good indicator that directly reflects cerebral function state. Dong et al. [50] did a series of experimental studies on the influence and bidirectional regulation of SA on the electric activities of pain reaction neurons in acute ICH rats. The results showed that nerve cell function in local, neighboring, and remote area of $\mathrm{ICH}$ lesion, and the opposite side of lesion was damaged in the early stage after ICH. The functional activity decreased and the dysfunction area was far more than haematoma and its peripheral area. At the early stage SA significantly reduced these injuries after ICH. In addition, SA has bidirectional and beneficial regulation effect on electric activity of pain-reaction neurons in experiment animals. Zhao et al. [51] reported that acupuncture on Baihui (GV 20) penetrating Taiyang (EX-HN5) could recover abnormal somatosensory-evoked potentials (SEPs) and improve coordination and compensation functions among cortical functional areas, thus improve the neurological function in acute ICH patients.

\section{Others}

Acupuncture on Baihui (GV 20) penetrating Qubin (GB 7) promoted the nerve growth factor (NGF) gene expression around the hematoma and had a role in neuroprotection in acute ICH rats [52]. In addition, acupuncture on Baihui (GV 20) penetrating Qubin (GB 7) promoted the expression of endogenous glial cell-line-derived neurotrophic factor (GDNF) in acute ICH rats [53]. Acupuncture on Baihui (GV 20) penetrating Taiyang (EX-HN5) could lower the level of serum S-100B, increase the level of insulin-like growth factor 1 (IGF-1), improve the nervous functional deficit, improve the coordination and compensation function, and improve the general nervous function between cortical functional areas, so as to improve activity of daily living in acute ICH patients $[54,55]$.

\section{Mechanism of Instant Effect}

Instant effect means that in 10 minutes after acupuncture, muscle force in paralysis side could elevate 2 grades or more. Inoue et al. [56] showed that SA indeed has rapid and powerful effects to remove limb paralysis caused either by cerebral infarct or by cerebral haemorrhage in rats. Dong et al. [57, 58] found that $60.71 \%(34 / 56) \mathrm{ICH}$ patients showed instant effect in SA group, while no case in drugtherapy and surgery haematoma aspiration group. This study showed exact instant effect of SA therapy. The mechanisms may be that SA improved the disorder of cerebral blood flow in ischemic region, or that it changed the excitability of cerebral cortex nerve cell and led to the retroconversion of the excitability of cerebral nerve cell that was depressed by the stimulation of hemorrhage or the oppression of haematoma. The generalization effect of projection capability disappeared and the nerve cell in shock or resting state after cerebral hemorrhage was awakened. Excitability of nervous cell recovered quickly and the cerebral compensation ability increased. The brain electric activity speeded up and might 
promote the conduction of central nervous system. This may be one of the mechanisms for the instant effect for SA. Wang et al. [59] showed that SA may regulate the electrophysiological activity of brain nerve cell and changed excitability of cerebral cortex nerve cell, thus awakened brain nerve cells that were in the state of shock or dormancy after ICH. Song et al. [60] indicated that the instant effect of SA treatment for $\mathrm{ICH}$ was through delaying the inhibition and death of neurons in the hematoma, relieving the inhibition of neuron discharge in the area surrounding hematoma, and reducing the influence on latency of evoked discharge. SA therapy modulated the state of neuronal function and promoted the recovery of neuronal function. Therefore, the existence of instant effect of SA illustrated the reliability of curative effect of SA on acute ICH, the importance of early treatment, and the scientific efficiency of region division and acupoint selection. The instant effect of SA, the possible mechanisms, and treatment course would be important questions for the study of SA therapy for ICH.

\section{Summary}

Recent animal and clinical studies have provided important information about the mechanisms of SA therapy for acute $\mathrm{ICH}$. Current evidences indicate that various mechanisms are associated with SA therapy for $\mathrm{ICH}$-induced brain injury. They are the influences on hematoma, brain edema- and $\mathrm{BBB}$, the products released from haematoma, the immune and inflammatory reaction, focal perihemorrhagic hypoperfusion and hemorheology, neuroelectrophysiology, and so on. A better understanding of the role of the products released from haematoma and their effect on SA therapy could have profound implications for treatment of ICH patients. Functional magnetic resonance imaging (fMRI) will contribute to revealing the therapeutic mechanisms of SA therapy for ICH in the future, but there are few studies in this field till now. In the near future, SA therapy could offer potential novel promise in the therapeutic approach to acute ICH. However, the clarification of the mechanisms of SA therapy for ICH is urgently needed for better guidance of the ICH treatment and improvement of the prognosis of patients.

\section{Authors' Contribution}

Z. Liu, L. Guan, and Y. Wang contributed equally to this work.

\section{Acknowledgment}

This paper is supported by the Project of Wenzhou Municipal Science and Technology Bureau in Zhejiang (Y20110031, Y20070038) and Administration of Traditional Chinese Medicine of Zhejiang Province (2011ZB094); the Project is supported by Open Research Fund of Zhejiang First-foremost Key Subject, Acupuncture and Tuina (ZTK2010A03).

\section{References}

[1] G. A. Donnan, M. Fisher, M. Macleod, and S. M. Davis, "Stroke," The Lancet, vol. 371, no. 9624, pp. 1612-1623, 2008.

[2] V. L. Feigin, "Stroke epidemiology in the developing world," The Lancet, vol. 365, no. 9478, pp. 2160-2161, 2005.

[3] A. Bhalla and D. Hargroves, "Does early medical intervention have a role in the management of intracerebral haemorrhage?" International Journal of Clinical Practice, vol. 62, no. 4, pp. 633-641, 2008.

[4] V. L. Feigin, "Stroke in developing countries: can the epidemic be stopped and outcomes improved?" Lancet Neurology, vol. 6, no. 2, pp. 94-97, 2007.

[5] B. Jiang, W. Z. Wang, H. Chen et al., "Incidence and trends of stroke and its subtypes in China: results from three large cities," Stroke, vol. 37, no. 1, pp. 63-68, 2006.

[6] G. A. Donnan, G. J. Hankey, and S. M. Davis, "Intracerebral haemorrhage: a need for more data and new research directions," The Lancet Neurology, vol. 9, no. 2, pp. 133-134, 2010.

[7] F. K. H. Sze, F. F. Yeung, E. Wong, and J. Lau, "Does Danshen improve disability after acute ischaemic stroke?" Acta Neurologica Scandinavica, vol. 111, no. 2, pp. 118-125, 2005.

[8] B. Wu, M. Liu, H. Liu et al., "Meta-analysis of traditional Chinese patent medicine for ischemic stroke," Stroke, vol. 38, no. 6, pp. 1973-1979, 2007.

[9] G. Q. Zheng and P. X. Huang, "Origin and development of hemorrhagic stroke," Chinese Journal of Medical History, vol. 35 , pp. 25-28, 2005.

[10] M. Liu, B. Wu, W. Z. Wang, L. M. Lee, S. H. Zhang, and L. Z. Kong, "Stroke in China: epidemiology, prevention, and management strategies," Lancet Neurology, vol. 6, no. 5, pp. 456-464, 2007.

[11] S. Lu, "Scalp acupuncture therapy and its clinical application," Journal of Traditional Chinese Medicine, vol. 11, no. 4, pp. 272280, 1991.

[12] G. Q. Zheng, "Methodological standards for experimental research on stroke using scalp acupuncture," Acupuncture \& Electro-Therapeutics Research, vol. 34, no. 1-2, pp. 1-13, 2009.

[13] G. Q. Zheng, Z. M. Zhao, Y. Wang et al., "Meta-analysis of scalp acupuncture for acute hypertensive intracerebral hemorrhage," Journal of Alternative and Complementary Medicine, vol. 17, pp. 293-2999, 2011.

[14] S. Y. Jin, Scalp Acupuncture Wall Chart (English-Chinese), People's Medical Publishing House, Beijing, China, 1st edition, 2006.

[15] S. F. Jiao, Scalp Acupuncture, Shanxi People' s Publishing House, Taiyuan, China, 1st edition, 1982.

[16] F. C. Wang, Chinese Alternative Treatment Methods-Scalp Acupuncture Therapy, People's Medical Publishing House, Beijing, China, 2nd edition, 2008.

[17] S. F. Chiao, "Scalp acupuncture in brain diseases," Chinese Medical Journal, vol. 3, no. 5, pp. 325-328, 1977.

[18] W. Wen, "Scalp acupuncture in China," American Journal of Chinese Medicine, vol. 5, no. 1, pp. 101-104, 1977.

[19] T. H. Liu and M. S. Sadove, "Scalp needle therapy: acupuncture treatment for central nervous system disorders," American Journal of Chinese Medicine, vol. 2, no. 3, pp. 261-269, 1974.

[20] M. Q. Zhu, Zhu's Scalp Acupuncture, Eight Dragons Publishing, Hong Kong, 1st edition, 1992.

[21] T. Yamamoto, "New scalp acupuncture," Acupuncture in Medicine, vol. 6, no. 2, pp. 46-48, 1989.

[22] T. Yamamoto and H. Yamamoto, Yamamoto New Scalp Acupuncture, Axel Springer Japan Publishing, Tokyo, Japan, 1st edition, 1998. 
[23] WHO Scientific Group on International Acupuncture Nomenclature, A Proposed Standard International Acupuncture Nomenclature: Report of a WHO Scientific Group, World Health Organization, Geneva, Switzerland, 1991.

[24] "Scalp acupuncture therapy (Terminology)," Journal of Acupuncture and Tuina Science, vol. 11, p. 14, 2003.

[25] G. Dong, L. Guan, F. Sun et al., "Comparative study on therapeutic effect of scalp-acupuncture in treating acute cerebral hemorrhage," International Journal of Clinical Acupuncture, vol. 3, pp. 31-38, 1992.

[26] J. Li, J. Xiao, and G. R. Dong, "Clinical study on effect of scalpacupuncture in treating acute cerebral hemorrhage," Chinese Journal of Integrated Medicine, vol. 5, pp. 265-268, 1999.

[27] G. Q. Zheng, Y. Wang, and X. T. Wang, "Literature foundation of scalp-acupuncture treatment of cerebral hemorrhage and its recent progress in clinical research," Acupuncture Research, vol. 31, pp. 181-184, 2006.

[28] A. I. Qureshi, A. D. Mendelow, and D. F. Hanley, "Intracerebral haemorrhage," The Lancet, vol. 373, no. 9675, pp. 1632-1644, 2009.

[29] J. M. Gebel Jr., E. C. Jauch, T. G. Brott et al., "Relative edema volume is a predictor of outcome in patients with hyperacute spontaneous intracerebral hemorrhage," Stroke, vol. 33, no. 11, pp. 2636-2641, 2002.

[30] J. W. Dong, C. L. Bao, and W. Z. Gong, "Clinical observation on effect of scalp penetration acupuncture in treating acute intracerebral hemorrhage," Clinical Journal of Traditional Chinese Medicine, vol. 18, pp. 341-342, 2006.

[31] W. Zou, G. W. Zhang, F. Liu et al., "Experimental study on the effect of acupuncturing Baihui penetrating Qubin on brain histomophology in rats with cerebral hemorrhage," Journal of Clinical Acupuncture and Moxibustion, vol. 23, pp. 41-44, 2007.

[32] W. B. Fu, L. Fan, and L. L. Mo, "Experimental study on acupuncture treatment of acute intracerebral hemorrhage in rats," Chinese Archive of Traditional Chinese Medicine, vol. 21, pp. 1787-1788, 2003.

[33] R. F. Keep, J. Xiang, S. R. Ennis et al., "Blood-brain barrier function in intracerebral hemorrhage," Acta Neurochirurgica, Supplementum, no. 105, pp. 73-77, 2008.

[34] X. J. Ji, H. He, Z. X. Wen et al., "Experimental study on the effect of scalp-acupuncture on BBB in acute cerebral hemorrhage rat," Chinese Journal of Critical Care Medicine, vol. 21, pp. 624-625, 2001.

[35] G. W. Zhang, W. Zou, and F. Liu, "Experimental study on the influence of Baihui-Qubin scalp acupuncture on expression of MMP-9 in rats of acute intracerebral hemorrhage," Chinese Practical Medicine, vol. 5, pp. 1-3, 2010.

[36] G. W. Zhang, W. Zou, and F. Liu, "Experimental study on the influence of Baihui-Qubin scalp acupuncture on AQP4 expression in rats of acute intercerebral hemorrhage," Information on Traditional Chinese Medicine, vol. 27, pp. 7579, 2010.

[37] H. Matsuoka and R. Hamada, "Role of thrombin in CNS damage associated with intracerebral haemorrhage: opportunity for pharmacological intervention?" CNS Drugs, vol. 16, no. 8, pp. 509-516, 2002.

[38] Q. B. Chi, L. Wang, and W. Zou, "Effect of acupuncture on the expression of ParPAR-1 in the brain tissue around hematoma of the rats with intercerebral hemorrhage," Journal of Clinical Acupuncture Moxibustion, vol. 26, pp. 48-50, 2010.

[39] J. Wang and S. Doré, "Inflammation after intracerebral hemorrhage," Journal of Cerebral Blood Flow and Metabolism, vol. 27, no. 5, pp. 894-908, 2007.
[40] F. Liu, G. W. Zhang, W. Zhou et al., "Influence of scalp acupuncture on expression of IL- $1 \beta$ after intracerebral hemorrhage in rats," Chinese Journal of Trauma and Disability Medicine, vol. 16, pp. 2-5, 2008.

[41] Y. Z. Zhao and C. D. Yu, "Effect of cranial needling combined with medicine on IL- 6 in the patient of acute cerebral hemorrhage," Chinese Acupuncture \& Moxibustion, vol. 28, no. 8, pp. 569-572, 2008.

[42] X. G. Yu, G. R. Dong, and J. H. Zhou, "Effect of acupuncture on TNF-? in the rat with acute cerebral hemorrhage," Chinese Acupuncture \& Moxibustion, vol. 24, pp. 403-406, 2004.

[43] W. Zou, R. X. Shi, and X. P. Yu, "The regulative effect of acupuncture on HSP70 mRNA expression in rats with acute hypertensive cerebral hemorrhage," Acta Chinese Medicine and Pharmacology, vol. 31, pp. 46-47, 2003.

[44] S. Y. Jiang, J. Du, and G. R. Dong, "Clinical immunological study on scalp acupuncture therapy for acute cerebral apoplexy," Heilongjiang Journal of Traditional Chinese Medicine, vol. 30, pp. 53-54, 2001.

[45] P. D. Schellinger, J. B. Fiebach, K. Hoffmann et al., "Stroke MRI in intracerebral hemorrhage: is there a perihemorrhagic penumbra?" Stroke, vol. 34, no. 7, pp. 1674-1679, 2003.

[46] C. L. Bao and H. S. Dong, "Effect of scalp-acupuncture on energy metabolism of brain tissue in intracerebral hemorrhage rats," Acupuncture Research, vol. 33, no. 2, pp. 93-97, 2008.

[47] C. L. Bao, J. Li, and H. S. Dong, "Effects of scalp electroacupuncture on cerebral Glut-1 and Glut-1 mRNA expressions in intracerebral hemorrhage rats," Acta Univ Tradit Med Sin Pharmacol Shanghai, vol. 22, pp. 50-54, 2008.

[48] C. L. Bao, H. S. Dong, G. R. Dong et al., "Effects of scalp penetration acupuncture on plasma endothelin (ET) and calcitonin related peptide (CGRP) contents in the patient of acute intracerebral hemorrhage," Chinese Acupuncture \& Moxibustion, vol. 25, pp. 717-719, 2005.

[49] C. L. Bao, L. R. Zhang, and G. R. Dong, "Effect of scalp penetration acupuncture on plasma level of ET-1 and NSE in patients with acute intracerebral hemorrhage," Journal of Clinical Acupuncture and Moxibustion, vol. 21, pp. 21-22, 2005.

[50] G. R. Dong, Y. Bai, C. Y. Jin, H. S. Dong, and L. Q. Li, "Bidirectional adjustment of scalp acupuncture to neuron electric-activity of rats with acute cerebral hemorrhage," Chinese Journal of Clinical Rehabilitation, vol. 10, no. 39, pp. 48-50, 2006.

[51] G. J. Zhao, Y. Li, Y. Chen et al., "Observation on somatosensory evoked potentials in acute intracerebral hemorrhage patients with scalp penetration acupuncture treatment," Journal of Clinical Acupuncture and Moxibustion, vol. 25, pp. 2627, 2009.

[52] F. Liu, W. Zou, and Z. R. Ni, "Effect of scalp penetration acupuncture on nerve growth factor gene expression in cerebral hemorrhage rats," Journal of Clinical Acupuncture and Moxibustion, vol. 23, pp. 46-49, 2007.

[53] G. W. Zhang, W. Zou, F. Liu et al., "Experimental study on the influence of acupuncture on GDNF expression in rats of acute intracerebral hemorrhage," Journal of Inner Mongolia University Nationalities Mar, vol. 25, pp. 191-193, 2010.

[54] J. J. Li and G. R. Dong, "Scalp acupoints penetration in treating acute hypertensive intracerebral hemorrhage and its correlativity with serum S100B protein and insulinlike growth factor-1," Journal of Sichuan Traditional Chinese Medicine, vol. 26, pp. 119-121, 2008. 
[55] J. J. Li and G. R. Dong, "Scalp acupoints penetration in treating acute hypertensive intracerebral hemorrhage and its correlativity with serum S100B protein," Journal of Acupuncture and Tuina Science, vol. 4, pp. 274-278, 2006.

[56] I. Inoue, L. Chen, L. Zhou, X. Zeng, and H. Wang, "Reproduction of scalp acupuncture therapy on strokes in the model rats, spontaneous hypertensive rats-stroke prone (SHR-SP)," Neuroscience Letters, vol. 333, no. 3, pp. 191-194, 2002.

[57] G. R. Dong, X. Zhang, D. Li et al., "Research on scalp acupuncture for acute hypertensive cerebral hemorrhage," Chinese Acupuncture \& Moxibustion, vol. 14, pp. 13-15, 2006.

[58] G. R. Dong, Z. Wang, and B. Z. Wu, "Discussion on the mechanism of immediate effect of head needling on acute encephalorrhagia," Chinese Acupuncture \& Moxibustion, vol. 14, pp. 26-28, 1994.

[59] Y. Q. Wang, C. F. Luo, and X. Y. Teng, "Effect of somatosensory evoked potential of rats with acute cerebral hemorrhage treated with scalp acupuncture," Information on Traditional Chinese Medicine, vol. 20, pp. 47-48, 2003.

[60] C. H. Song, W. Z. Zhu, and F. C. Zhang, "Experimental study on the influence of scalp acupuncture on neuron discharge in ICH rat," Chinese Journal of Traditional Medicine Science Technology, vol. 10, no. 4, p. 222, 2003. 


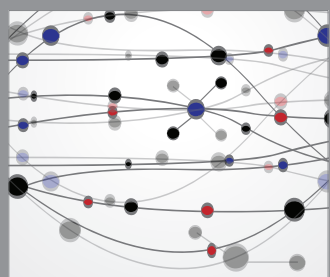

The Scientific World Journal
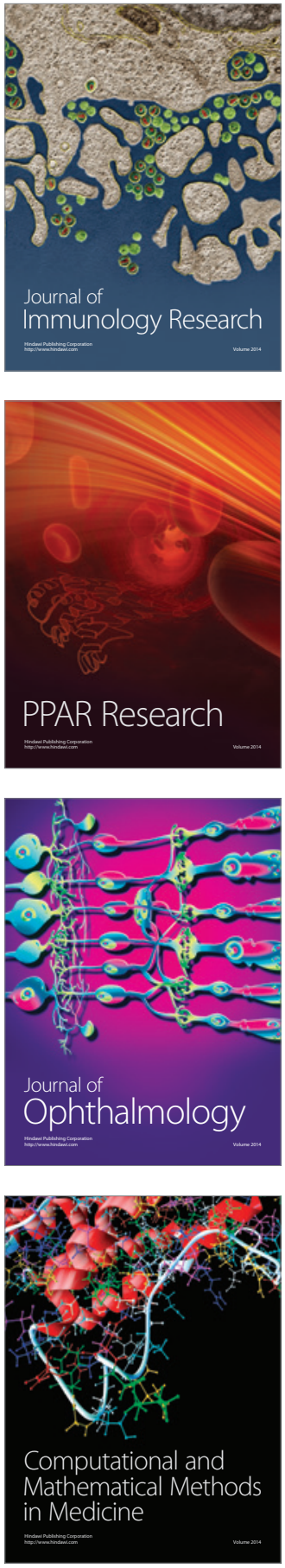

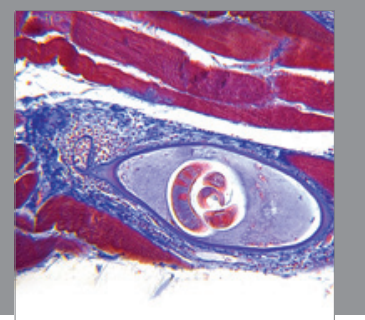

Gastroenterology

Research and Practice
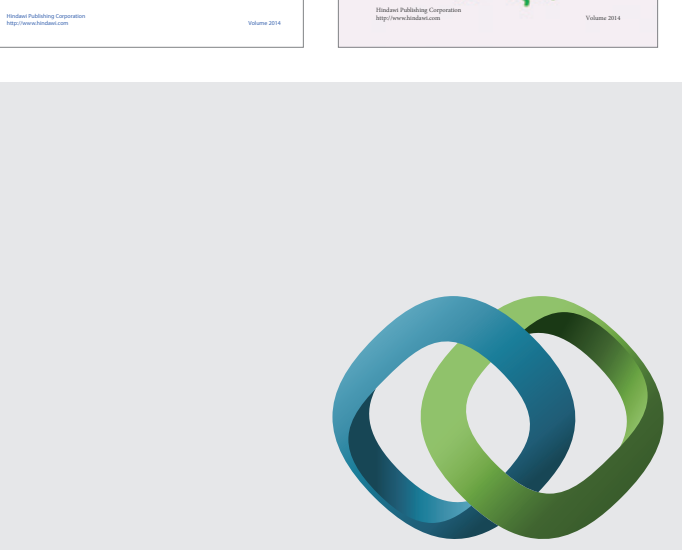

\section{Hindawi}

Submit your manuscripts at

http://www.hindawi.com
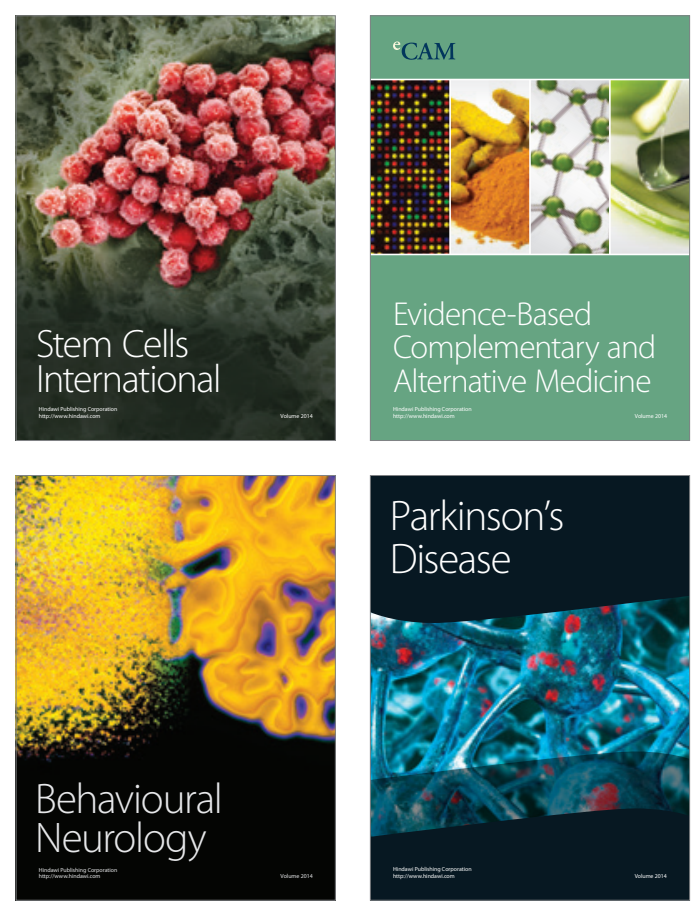

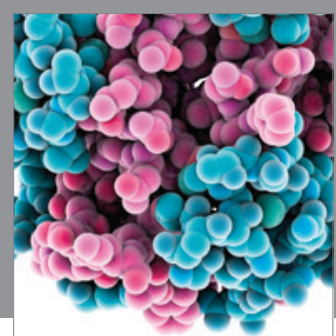

Journal of
Diabetes Research

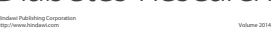

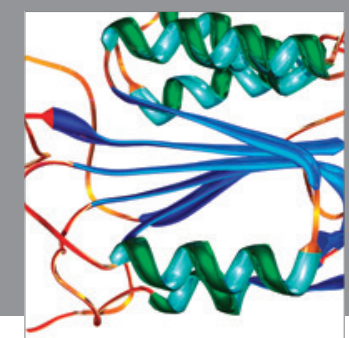

Disease Markers
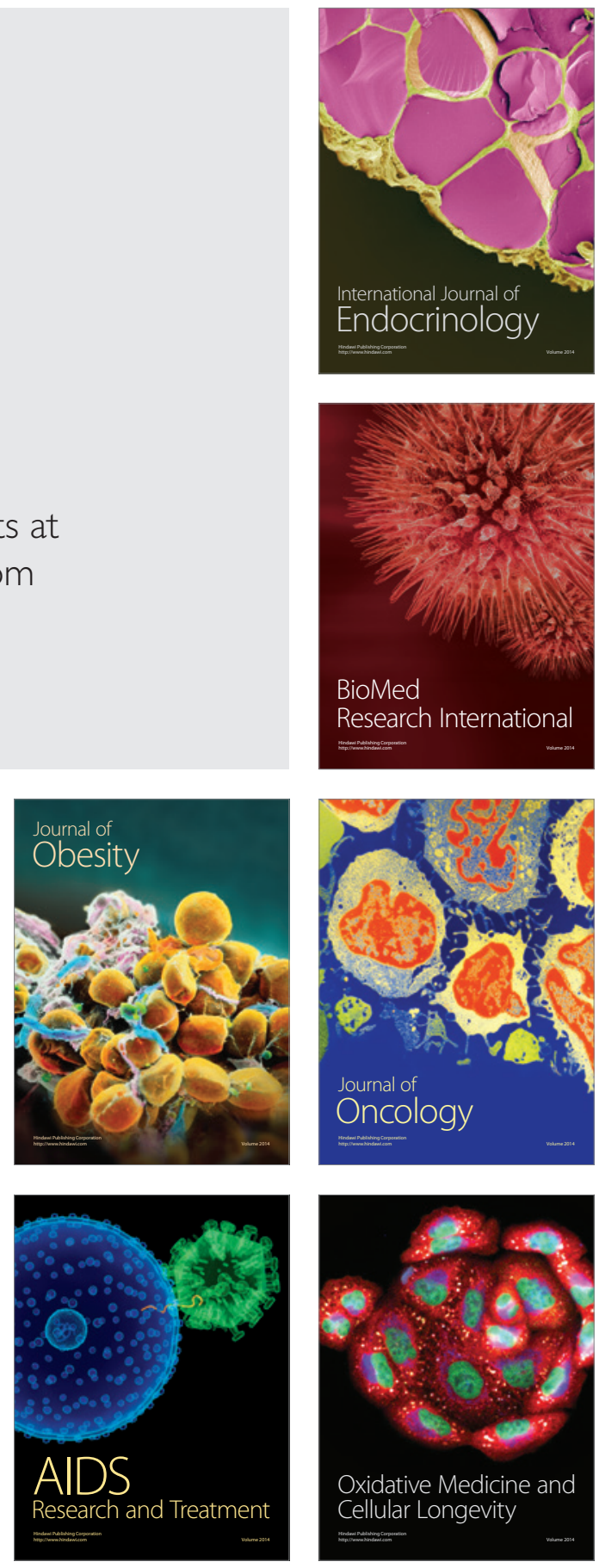